\title{
Radiation Induced Baroreflex Failure in Head and Neck Cancer Patients
}

\author{
Manikandan Abirami*, Subramaniyam Velkumary \\ Department of Physiology, JIPMER, Puducherry, INDIA. \\ *Correspondence \\ Dr. Manikandan Abirami. \\ Junior Resident, Department of Physiology, JIPMER, Puducherry, INDIA. \\ Phone: +91 9442300996 \\ Email: abiramimanikandan30@gmail.com \\ DOI : 10.5530/ijcep.2021.8.3.32
}

\section{NEWS}

Head and Neck Cancer (HNC) is the sixth most common cancer worldwide. In developing countries like India, $60 \%$ to $80 \%$ of cases are diagnosed only in advanced stage. The vital structures of head and neck region are infiltrated by the malignant cells, interfering with normal functions, like swallowing, breathing etc. ${ }^{[1]}$ Therefore, these patients are treated aggressively, with combined chemotherapy and radiotherapy (RT). Neck radiation has emerged as the most extensively used treatment modality, when vital organ preservation is considered. Ionizing waves used in RT, generates Reactive Oxygen Species (ROS) and free radicals. ROS are not only responsible for arresting the proliferation and killing of cancer cells, but also cause radiation -induced long-term complications. ${ }^{[2]}$

The incidence rate of late complications like Baroreflex failure and cardiovascular autonomic dysfunctions have tremendously increased over the past few years, due to advancement in treatment modality, increased survival rate, clinical awareness of radiation injury. Several mechanisms have been postulated for radiation induced baroreflex failure. The ROS leads to chronic inflammation of the irradiated neck region results in carotid artery fibrosis, causing baroreceptors malfunction. ${ }^{[3]}$ In addition to this, the afferent nerves coursing from the baroreceptors are damaged leading to baroreflex failure and altered cardiovascular autonomic function. Reduction in tonic inhibitory input to the vasomotor center, causes increased sympathetic outflow, resulting in impaired control of blood pressure and heart rate. ${ }^{[4]}$

\section{VIEWS}

\section{Parasymapthetic activity improves survival in HNC patients}

Post-irradiation baroreflex failure has wide range of clinical presentations from orthostatic hypotension to neurogenic supine hypertension. Previous studies with conventional cardiac autonomic function test (CAFT) in HNC patients, Correlated Impaired Vagal activity with poor prognosis. In our pilot study, CAFT was done before the onset of RT and the 6 months after the completion of RT. During followup, nearly half of the patients got expired. Post RT CAFT in the survivors, revealed improved cardiovagal activity. Improved vagal activity may be ascribed to activation of cholinergic anti-inflammatory pathways, in response to persistent inflammation induced by the cancer per se and post radiotherapy. As a result, there is an activation of efferent vagal nerve. The activated vagus nerve, inhibits the oxidative stress, inflammtion and symapthetic activity and improves the prognosis and survival in cancer patients.

\section{REFERENCES}

1. Pai SI, Westra WH. Molecular pathology of head and neck cancer: Implications for diagnosis, prognosis, and treatment. Annu Rev Pathol. 2009;4:49-70. doi: 10.1146/ annurev.pathol.4.110807.092158, PMID 18729723.

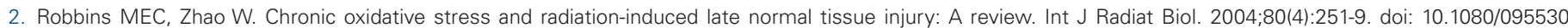
00410001692726 , PMID 15204702.

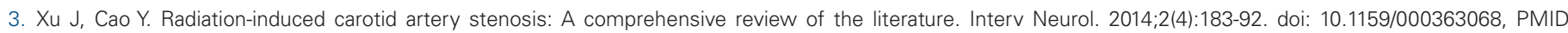
25337087.

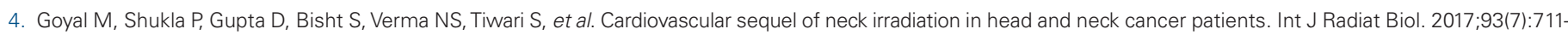
6. doi: 10.1080/09553002.2017.1303217, PMID 28376642

Cite this article: Abirami M, Velkumary S. Radiation Induced Baroreflex Failure in Head and Neck Cancer Patients. Int J Clin Exp Physiol. 2021;8(3):130. 